\title{
VOLUMES OF FLOWS
}

\author{
DAVID L. JOHNSON
}

(Communicated by David G. Ebin)

\begin{abstract}
If $F$ is an oriented nonsingular flow on a Riemannian manifold $M$, the volume of $F$ is defined as the $n$-dimensional measure of the unit vector field tangent to $F$, as a section of $T_{*}(M)$ with the induced metric. It is shown that, for any metric of the two-dimensional torus, and for any homotopy class of flows on the torus, there is a unique smooth flow of minimal volume within the homotopy class. It has been shown that the Hopf foliation on the round threesphere absolutely minimizes the volume of flows on $S^{3}$. In higher dimensions this is not the case; the Hopf fibrations are not even local minima of the volume functional for flows on the round five-sphere. It is not known whether a volume-minimizing flow on $S^{5}$ exists.
\end{abstract}

0. Introduction. The problem we will be considering is an extremely natural one: Any transversely-oriented, codimension- $q$ foliation $\mathscr{F}$ on a Riemannian manifold $M$ defines a section of the Grassmann bundle of $n-q$ planes tangent to $M, G_{\mathscr{F}}: M \rightarrow G\left(n-q, T_{*}(M)\right)$, by mapping $x \in M$ to $G_{\mathscr{F}}(x):=\mathscr{F}_{x} \subset T_{*}(M)$. $G\left(n-q, T_{*}(M)\right)$ has a natural metric induced from the metricon $M$ and the Riemannian connection. In this setting the image of $G_{\mathscr{F}}$ is thought of as the graph of the foliation $\mathscr{F}$. A natural question, due to H. Gluck and W. Ziller [7], to ask about such foliations is the following: which foliation is most efficient, in that its graph has minimal volume ( $n$-dimensional measure)?

In this article we will be concerned only with the case of flows, or one-dimensional foliations. The general case will be considered elsewhere.

If $\mathscr{F}$ is an oriented flow on a manifold $M$, with unit vector field $\xi$, then the volume of the flow is given by:

$$
\begin{aligned}
\int_{M}\left(1+\sum_{j}\left|\nabla_{e^{j}} \xi\right|^{2}\right. & +\sum_{j_{1}<j_{2}}\left|\nabla_{e^{j_{1}}} \xi \wedge \nabla_{e^{j_{2}}} \xi\right|^{2}+\cdots \\
& \left.+\sum_{j_{1}<\cdots<j_{n-1}}\left|\nabla_{e^{j_{1}}} \xi \wedge \cdots \wedge \nabla_{e^{j_{n-1}}} \xi\right|^{2}\right)^{1 / 2} d V
\end{aligned}
$$

for any orthonormal basis $\left\{e^{j}\right\}$ of $T_{*}(M)$. The top ( $n$ th) wedge will vanish since $\xi$ has values in the unit sphere bundle. The metric on the tangent bundle used here is defined by Sasaki in [15], and is the natural metric on $T_{*}(M)$ induced from the Riemannian metric on $M$.

Received by the editors September 10, 1987. Presented to the Society at the January, 1987 meeting in San Antonio.

1980 Mathematics Subject Classification (1985 Revision). Primary 58F18; Secondary 58E30, 58F 18, 58F25. 
Note that there is a trivial absolute minimum of the volume integrand when $\xi$ is parallel. That will usually not be possible, since that is equivalent to $\mathscr{F}$ and the orthogonal distribution $\mathscr{H}$ being totally geodesic.

In the following sections, special cases of the variational problems involved in minimizing the functional are considered, and the variations are derived using the specific properties of each case.

1. Flows on surfaces. If $\mathscr{F}$ is a flow on a compact surface $M$, which must be topologically a torus, then the graph $G_{F}$ is just a choice of unit vector field $\xi$ tangent to $\mathscr{F}$. The integrand which gives the area of the unit vector field $\xi$ is $\left(1+\left|\nabla_{e^{1}} \xi\right|^{2}+\left|\nabla_{e^{2}} \xi\right|^{2}\right)^{1 / 2} d V$, which can be computed in terms of any orthonormal basis of tangents at $x \in M$; take a unit vector field $\{X\}$ normal to the leaves, and the vector field $\xi$ itself. Thus the integrand is $\left(1+\left|\nabla_{X} \xi\right|^{2}+\left|\nabla_{\xi} \xi\right|^{2}\right)^{1 / 2} d V$. The terms represent the square of the norm of the second fundamental form of the orthogonal distribution, which for curves is the square of the (geodesic) curvature, plus the square of the geodesic curvature of the flow.

The homotopy classes, or path-components, in the space of all flows on a torus are given by the net number of oriented Reeb components in the flow, with Reeb components having different orientations counted with opposite signs. The main result of this section (below) locates a minimum-volume representable within each such class.

THEOREM 1.1. For any homotopy class of flows on a two-dimensional torus $M$ with any given Riemannian metric, there is a smooth flow $\mathscr{F}$ of smallest area, unique up to rotation by a constant angle.

PROOF. Let $\left\{e^{1}, e^{2}\right\}$ be a chosen orthonormal framing of $T_{*}(M)$. Any flow $\mathscr{F}$ can then be described, in terms of a unit vector field $\xi$ tangent to $\mathscr{F}$, by $\xi=\cos (\theta) e^{1}+\sin (\theta) e^{2}$, where $\theta: M \rightarrow \mathbf{R} / 2 \pi \mathbf{Z} \simeq S^{\mathbf{1}}$. Conversely, any such function determines $\mathscr{F}$, and homotopy classes of foliations correspond to homotopy classes of such maps, that is, \{homotopy classes of foliations $\simeq \simeq \pi^{1}(M) \simeq H_{1}(M, \mathbf{Z}) \simeq$ $H^{1}(M, \mathbf{Z})$. The volume integrand then becomes

$$
\left(1+\left(\left\langle\nabla_{e^{1}} e^{1}, e^{2}\right\rangle+e^{1}(\theta)\right)^{2}+\left(\left\langle\nabla_{e^{2}} e^{1}, e^{2}\right\rangle+e^{2}(\theta)\right)^{2}\right)^{1 / 2} d V .
$$

If $\alpha(A)=\left\langle\nabla_{A} e^{1}, e^{2}\right\rangle$, then the integrand simplifies to $\left(1+|\alpha+d \theta|^{2}\right)^{1 / 2} d V$. Now,

$$
d \alpha\left(e^{1}, e^{2}\right)=e^{1}\left\langle\nabla_{e^{2}} e^{1}, e^{2}\right\rangle-e^{2}\left\langle\nabla_{e^{1}} e^{1}, e^{2}\right\rangle-\left\langle\nabla_{\left[e^{1}, e^{2}\right]} e^{1}, e^{2}\right\rangle d V=K d V,
$$

where $K$ is the Gaussian curvature, since $\left\langle\nabla_{A} e^{1}, \nabla_{B} e^{2}\right\rangle=0$. Also, $d \theta=h+d f$, where $h$ is the harmonic representative of the cohomology class of the difference between the homotopy classes of $\mathscr{F}$ and the $e^{1}$-flow. Choosing $\theta$ so that $d \theta=$ $-h_{\alpha}+d g$, where $\alpha=h_{\alpha}+d g+* d \sigma$ is the orthogonal decomposition of $\alpha$ into exact, coexact, and harmonic pieces, there is a flow $\mathscr{F}_{0}$ for which $\alpha+d \theta=* d \sigma$, where $\sigma$ is the unique function orthogonal to the constants so that $\Delta \sigma=-* d * d \sigma=K$.

Replace $\left\{e^{1}, e^{2}\right\}$ by $\{\xi, X\}$ for the foliation $\mathscr{F}_{0}$, so it may be assumed that $\alpha=$ $\left\langle\nabla e^{1}, e^{2}\right\rangle=* d \sigma$. Then, for any $\mathscr{F}$ with unit tangent field $\xi=\cos \theta e^{1}+\sin \theta e^{2}$, the volume integrand becomes $\left(1+|h+d f+* d \sigma|^{2}\right)^{1 / 2} d V$. The task of minimizing volume within a given homotopy class of flows on $M$ then reduces to minimizing

$$
F(f)=\int_{M}\left(1+|h+d f+* d \sigma|^{2}\right)^{1 / 2} d V
$$


over $f \in C^{\infty}(M)$.

If $f$ is a critical point of $F(f)$, then for all $g \in C^{\infty}(M)$,

$$
\begin{aligned}
0 & =\frac{d}{d t} \mid 0_{\mid 0} F(f+t g)=\int_{M}\langle d g, h+d f+* d \sigma\rangle\left(1+|h+d f+* d \sigma|^{2}\right)^{-1 / 2} d V \\
& =\int_{M}\left\langle g, d^{*}\left((h+d f+* d \sigma)\left(1+|h+d f+* d \sigma|^{2}\right)^{-1 / 2}\right)\right\rangle d V,
\end{aligned}
$$

so the critical equation becomes $0=d^{*}\left((h+d f+* d \sigma)\left(1+|h+d f+* d \sigma|^{2}\right)^{-1 / 2}\right)$, equivalently,

$$
0=d * d f+\frac{1}{2}(* h+* d f-d \sigma) \wedge\left(d|h+d f+* d \sigma|^{2}\right)\left(1+|h+d f+* d \sigma|^{2}\right)^{-1} .
$$

LEMMA 1.2. The Euler-Lagrange equation for the volume functional $F(f)$ of a flow on a surface is an elliptic, semilinear equation.

ProOF. Set $u=(* h+* d f-d \sigma)\left(1+|h+d f+* d \sigma|^{2}\right)^{-1 / 2} \cdot|u|^{2}<1$, pointwise. At the center of a Riemann normal coordinate system, where $g_{i j}=\delta_{i j}, \partial g_{i j} / \partial x_{k}=0$, if $h=a_{i} d x^{i}$ and $u=u_{i} d x^{i}$, then:

$$
\begin{aligned}
d|h+d f+* d \sigma|^{2}=2 & {\left[\left(a_{1}+\frac{\partial f}{\partial x^{1}}-\frac{\partial \sigma}{\partial x^{2}}\right)\left(\frac{\partial a_{1}}{\partial x^{i}}+\frac{\partial^{2} f}{\partial x^{1} \partial x^{i}}-\frac{\partial \sigma}{\partial x^{2} \partial x^{i}}\right)\right.} \\
& \left.+\left(a_{2}+\frac{\partial f}{\partial x^{2}}+\frac{\partial \sigma}{\partial x^{1}}\right)\left(\frac{\partial a_{2}}{\partial x^{i}}+\frac{\partial^{2} f}{\partial x^{2} \partial x^{i}}+\frac{\partial^{2} \sigma}{\partial x^{1} \partial x^{i}}\right)\right] d x^{i} .
\end{aligned}
$$

The Euler-Lagrange equation then becomes

$$
\begin{aligned}
0= & \frac{\partial^{2} f}{\left(\partial x^{1}\right)^{2}}\left(1-u_{2}^{2}\right)+\frac{\partial^{2} f}{\left(\partial x^{2}\right)^{2}}\left(1-u_{1}^{2}\right)+2 u_{1} u_{2} \frac{\partial^{2} f}{\partial x^{1} \partial x^{2}} \\
& +\left(u_{1} u_{2}\right)\left(\frac{\partial a_{1}}{\partial x^{2}}-\frac{\partial^{2} \sigma}{\partial x^{2} \partial x^{2}}\right)+\left(-u_{1}^{2}\right)\left(\frac{\partial a_{2}}{\partial x^{2}}+\frac{\partial^{2} \sigma}{\partial x^{1} \partial x^{2}}\right) \\
= & \left(u_{2}^{2}\right)\left(\frac{\partial a_{1}}{\partial x^{1}}-\frac{\partial \sigma}{\partial x^{2} \partial x^{1}}\right)+\left(u_{1} u_{2}\right)\left(\frac{\partial a_{2}}{\partial x^{1}}+\frac{\partial^{2} \sigma}{\partial x^{1} \partial x^{1}}\right) .
\end{aligned}
$$

Since $\xi_{1}^{2}\left(1-u_{2}^{2}\right)+\xi_{2}^{2}\left(1-u_{1}^{2}\right)+2 \xi_{1} \xi_{2} u_{1} u_{2}$ is positive-definite for $|u|<1$, the equation is elliptic.

LEMMA 1.3. There is a unique first-order linear map

$$
H: E^{1}(M) \rightarrow \operatorname{Hom}\left(E^{1}(M), E^{1}(M)\right)
$$

so that, for all $\alpha, \beta \in E^{1}(M), d(*(\alpha, \beta))=H_{\alpha}(\beta)-H_{\beta}(\alpha)$. Moreover, $H_{f \alpha}(\beta)=$ $(*(\alpha \wedge \beta)) d f+f H_{\alpha}(\beta)$, and $H_{* \alpha}(* \beta)=H_{\alpha}(\beta)$.

PROOF. Compute in terms of a Riemann normal coordinate system centered at $x \in M$. (This will not be independent of coordinates, but will be independent of choice of Riemann normal coordinates, since any two will only differ at $x$ by a rotation with vanishing derivatives at $x$.) Then, for $\alpha=a_{i} d x^{i}, \beta=b_{i} d x^{i}$,

$$
d(*(\alpha \wedge \beta))=\left(\frac{\partial a_{1}}{\partial x^{i} b_{2}}-\frac{\partial a_{2}}{\partial x^{i} b_{1}}\right) d x^{i}-\left(\frac{\partial b_{1}}{\partial x^{i} a_{2}}-\frac{\partial b_{2}}{\partial x^{i} a_{1}}\right) d x^{i}
$$

so setting

$$
H_{\alpha}=\left(\frac{\partial a_{1}}{\partial x^{i}} \frac{\partial}{\partial x^{2}}-\frac{\partial a_{2}}{\partial x^{i}} \frac{\partial}{\partial x^{1}}\right) \otimes d x^{1}
$$


with respect to this coordinate system at the center gives a well-defined operator. It is easy to see that this definition is independent of choice of Riemann normal coordinates, and if $\alpha \in \mathscr{H}_{s}$, the Sobolev space of functions with square-integrable derivatives of order $\leq s$, then $H_{\alpha} \in \mathscr{H}_{s-1}\left(T_{*}(M) \otimes T^{*}(M)\right)$. The last statements are trivial.

This lemma easily implies that $d\left(|\alpha|^{2}\right)=d(*(\alpha \wedge * \alpha))=H_{\alpha}(* \alpha)-H_{* \alpha}(\alpha)=$ $2 H_{\alpha}(* \alpha)$, if $M$ is a two-manifold. Setting $\alpha=h+d f+* d \sigma$, the Euler-Lagrange equation of the volume functional, from the above, may be re-expressed as follows, where $u=* \alpha\left(1+|\alpha|^{2}\right)^{-1 / 2}$ as before:

$$
\begin{aligned}
0 & =d * d f+\frac{1}{2}(* h+* d f-d \sigma) \wedge\left(d|h+d f+* d \sigma|^{2}\right)\left(1+|h+d f+* d \sigma|^{2}\right)^{-1} \\
& =d * d f+u \wedge H_{\alpha}(* \alpha)\left(1+|h+d f+* d \sigma|^{2}\right)^{-1 / 2} \\
& =d * d f+u \wedge H_{\alpha}(u)=d * d f+u \wedge H_{d f}(u)+u \wedge H_{h+* d \sigma}(u) .
\end{aligned}
$$

Consider the elliptic linear operator $L: \mathscr{H}_{s}(M) \rightarrow \mathscr{H}_{s-2}(M)$ given by $L(y)=$ $* d * d y+*\left(u \wedge H_{d y}(u)\right)+*\left(u \wedge H_{h+* d \sigma}(u)\right)$, where $u$ is defined as above for a fixed $f \in \mathscr{H}_{s}(M)$.

LEMMA 1.4. $L(y)=0$ has a unique solution $y \in \mathscr{H}_{s+1}$ orthogonal to the space of constant functions.

PROOF. Existence and regularity of the solutions are standard linear elliptic theory. To show uniqueness, note that the difference of any two solutions gives a solution $z$ of $* d * d z+*\left(u \wedge H_{d z}(u)\right)=0$. Assume that $s$ is large enough so that $z \in C^{3}(M)$. Near a maximum of $z$, the hessian $\operatorname{Hess}(z)$ will be negative semidefinite. At any point $p$ sufficiently near the maximum, pick a Riemann normal coordinate system centered at $p$ so that $u=a d x^{1}$ at $p$, with $0<a<1(|u|<1)$. Then

$$
\begin{aligned}
0 & =\frac{\partial^{2} z}{\left(\partial x^{1}\right)^{2}}+\frac{\partial^{2} z}{\left(\partial x^{2}\right)^{2}}+*\left(a d x^{1} \wedge\left(\frac{-\partial^{2} z}{\partial x^{2} \partial x^{i} a}\right) d x^{i}\right) \\
& =\frac{\partial^{2} z}{\left(\partial x^{1}\right)^{2}}+\left(1-a^{2}\right) \frac{\partial^{2} z}{\left(\partial x^{2}\right)^{2}} .
\end{aligned}
$$

But, since $\operatorname{Hess}(z)$ is negative semidefinite, and $a^{2}<1, \Delta z=0$ in this neighborhood. The maximum principle then implies that $z$ is constant.

Now consider the mapping $K: \mathscr{H}_{s} \rightarrow \mathscr{H}_{s}$ defined by $K(f)=y, y$ the unique solution orthogonal to the constants of $L(y)=0$. Since $K(f) \in \mathscr{H}_{s+1} \subset \mathscr{H}_{s}$, and $H_{s+1} \subset H_{s}$ is compactly embedded, $K$ is a compact operator. A solution of $0=G(f)=f-K(f)$ will be a solution of the Euler-Lagrange equation of the volume functional, and will necessarily be $C^{\infty}$ by Lemma 1.4. Such a solution exists if there is a bounded domain $\Omega \subset \mathscr{H}_{s}(M)$ so that the Leray-Schauder degree $d(G, 0, \Omega)$ is nonzero and $G \neq 0$ on $\partial \Omega[1]$. Choose a one-parameter family $g_{t}$ of Riemannian metrics on $M$, smooth in $t$, so that $g_{1}$ is the given metric, and $g_{0}$ is flat. Choose $A$ so that $A>2 \operatorname{Sup}_{t}\left\{\operatorname{Inf}\left\{\operatorname{Vol}_{g_{t}}(\mathscr{F}) \mid \mathscr{F}\right.\right.$ is in the fixed homotopy class of Theorem $2.3\}\}$, and also take $A>2 \operatorname{Sup}_{t}\left\{F_{t}(0)\right\}$, where $F_{t}$ is defined as above for the metric $g_{t}$. Set $\Omega=\left\{f \in L_{2}(M) \mid F_{t}(f) \leq A\right.$, for some $\left.t \in[0,1]\right\}$, where $F_{t}$ is the volume functional for the metric $g_{t}$. Since $F(r f)=\int_{M}\left(1+|h+r d f+* d \sigma|^{2}\right)^{1 / 2} d V$, which is asymptotic to $r \int_{M}|d f| d V \geq C r\left(\int_{M}|f|^{2} d V\right)^{1 / 2}$ for $r$ large, for some constant $C$ (by the Sobolev inequalities), $\Omega$ is bounded in $L_{2}[1]$. Since $\partial \Omega=\left\{f \mid F_{t}(f) \geq A\right.$, $F_{t}(f)=A$ for some $\left.t\right\}$, the infimum of $F_{t}$ will not lie on $\partial \Omega$ for any $t \in[0,1]$. 
Let $K_{t}: L_{2} \rightarrow L_{2}$ be the compact operator above defined for the metric $g_{t}$. $K_{t}(f) \neq f$ for $f \in \partial \Omega$, and $\left\{K_{t}(f) \mid f \in \bar{\Omega}, t \in[0,1]\right\}$ has compact closure since the mapping $K: \mathscr{H}_{s} \times I \rightarrow \mathscr{H}_{s+1}$ defined by $K(f, t)=K_{t}(f)$ is continuous, so that the bounded set $\Omega \times I$ has bounded image in $\mathscr{H}_{s+1}$, thus in $\mathscr{H}_{s}$ the image has compact closure. Thus, by [12], the degree $d(G, 0, \Omega)=d\left(G_{1}, 0, \Omega\right)$ is the same as $d\left(G_{0}, 0, \Omega\right)$. It can be seen that in the flat case, where $\sigma=0$ and $|h|^{2}$ is constant, $F(f)$ is minimized precisely when $d f=0$. Note that, since harmonic forms $h$ with respect to a Riemann normal coordinate system on the flat torus are of the form $h=a_{1} d x^{1}+a_{2} d x^{2}$ with $a_{i}$ constant, $H_{h+* d \sigma}=H_{h}=0$. The claim that $f$ is necessarily constant then follows from Lemma 1.4 .

$d\left(G_{0}, 0, \Omega\right)=d\left(G_{0}, 0, O_{\varepsilon}\right)$, where $O_{\varepsilon}$ is a small ball of radius $\varepsilon$ centered at 0, since $G_{0}(f)=0$ has only the one isolated solution, 0 [1]. However, since $H_{h}=0$, $K(f)=0$ for all $f$, so $G_{0}=\operatorname{Id}$, and thus $d(G, 0, \Omega)=1$. Thus $G(f)=0$ has a solution in $\Omega$, which shows that the volume functional has a critical point for any homotopy class.

To show that this critical point is unique, and is an absolute minimum within the homotopy class, it suffices to show that there is a two-form on the unit tangent bundle $T_{0}(M)$ calibrating the graph $\xi$ if and only if $\xi$ is a solution to the EulerLagrange equations of the volume functional [7].

Any section $\xi$ determines a flat connection $\omega$ on $T_{0}(M)$, considered as a principal $S^{1}$-bundle over $M$, by the condition that $\xi(M)$ be horizontal. The surface $\xi(M) \subset$ $T_{0}(M)$ extends to a $\omega$-horizontal foliation by the action of the group, which acts by isometries. The unit normal field of this foliation is divergence-free precisely when the leaves are minimal, that is, when $\xi$ satisfies the Euler-Lagrange equations of the volume functional. Since the two-form $\alpha=* \omega /|\omega|$ (pointwise norm) is dual to the normal field, it will be closed precisely when the foliation is minimal, and since its comass is one, and $\alpha\left(T_{*}(\xi(M))\right) \equiv 1$, when $d \alpha=0$ it will calibrate precisely $\xi$ and its translates under the action of the group. Thus, each critical point of the volume functional is calibrated, and so absolutely volume minimizing within its homology (homotopy) class.

The author would like to thank the referee for suggesting several improvements of this proof, particularly the final argument.

2. Hopf fibrations. Consider now the special cases of the Hopf fibrations $S^{1} \rightarrow S^{2 n+1} \rightarrow \mathbf{C P}^{n}$. For $n=1, \mathrm{H}$. Gluck and W. Ziller [7] have shown that the Hopf fibration of the round $S^{3}$ is the flow with least volume, using calibrated geometries. They point out, however, that their techniques break down for $n \geq 2$, in that the corresponding calibration does not have the Hopf flow as the minimum within its homology class in the unit tangent bundle. It is not known whether the minimum achieved by the calibration corresponds to the graph of a flow on $S^{2 n+1}$.

In this section their result is extended using direct methods. It is shown that, in contrast to the first case, the Hopf flow on $S^{5}$ is not even a local minimum of the volume functional. For all $n$, it is clear that the Hopf flows are critical flows of the volume functional.

Let $F(t)$ be the function $\operatorname{Vol}\left(\mathscr{F}_{t}\right)$, where $\mathscr{F}_{t}$ is a deformation of the Hopf fibration on $M=S^{5}$, and set $A=\mathscr{F}^{\prime}=h(\xi)[\mathbf{9}, \mathbf{1 0}]$. As shown in [10], $A$ is an arbitrary horizontal (perpendicular to $\xi$ ) vector field. 
Let $p \in \mathbf{C P}^{2}$, and choose a local framing $\left\{f^{2}, \ldots, f^{5}\right\}$ so that $\nabla_{f^{i}} f^{j}=0$. This can be accomplished by choosing a section $\sigma$ of the frame bundle over some neighborhood of $p$ with $\sigma(p)=\left\{f^{2}, \ldots, f^{5}\right\}$ and $\sigma_{*}\left(T_{*}\left(\mathbf{C P}^{2}, p\right)\right)=H=\operatorname{ker}(\omega)$, $\omega$ being the Riemannian connection. It may further be assumed that this framing is unitary, i.e., $J f^{2}=f^{3}, J f^{4}=f^{5}$, throughout the neighborhood $U$.

Now let $e^{1}=\xi$ be the unit tangent field to the Hopf fibration $\mathscr{F}$, and set $\left\{e^{2}, \ldots, e^{5}\right\}$ to be the horizontal $\left(\mathscr{F}^{\perp}\right.$ ), basic (in the sense of foliations, $[\mathbf{1 1}, \mathbf{1 4}]$ ) vector fields in $V=\pi^{-1}(U)$ which are $\pi$-related to $\left\{f^{2}, \ldots, f^{5}\right\}$, the basic lifts of those fields. In that case, $\left[e^{1}, e^{j}\right]$ will always be vertical, and $\nabla_{e^{i}} e^{j}, i, j \geq 2$ has horizontal portion the basic lift of $\nabla_{f^{i}} f^{j}[\mathbf{1 1}, \mathbf{1 4}]$. Along the fiber $\pi^{-1}(p)$, then, $\nabla_{e^{i}} e^{j}$ will be vertical, for $i, j \geq 2, \nabla_{e^{i}} e^{j}=\lambda_{i}^{j} e^{1}$. Then

$$
\left\langle\nabla_{e^{i}} e^{1}, e^{j}\right\rangle=-\left\langle e^{1}, \nabla_{e^{i}} e^{j}\right\rangle=-\lambda_{i}^{j}, \quad\left\langle\nabla_{e^{i}} e^{1}, e^{1}\right\rangle=0
$$

so $\nabla_{e^{i}} e^{1}=-\lambda_{i}^{j} e^{j}$. Since $\left[e^{1}, e^{i}\right]$ is vertical, and $\left\langle\nabla_{e^{1}} e^{i}, e^{1}\right\rangle=-\left\langle e^{i}, \nabla_{e^{1}} e^{1}\right\rangle=0$, $\nabla_{e^{1}} e^{i}=-\lambda_{i}^{j} e^{j}=\nabla_{e^{i}} e^{1}$. Since $\lambda_{j}^{i}=\frac{1}{2} \Omega\left(e^{i}, e^{j}\right)$, where $\Omega$ is the curvature of the canonical connection on the universal bundle over $\mathbf{C P}^{2}$, which is a lift of the Kähler form on $\mathbf{C P}^{2},\left\{f^{2}, \ldots, f^{5}\right\}$, hence $\left\{e^{2}, \ldots, e^{5}\right\}$, may be chosen so that $\nabla_{e^{2}} e^{3}=e^{1}=$ $-\nabla_{e^{3}} e^{2}, \nabla_{e^{4}} e^{5}=e^{1}=-\nabla_{e^{5}} e^{4}$, and all others vanish.

Along this chosen fiber, then, we have the following table of covariant derivatives:

$$
\begin{aligned}
& \nabla_{e^{1}} e^{1}=0, \quad \nabla_{e^{1}} e^{2}=-e^{3}=\nabla_{e^{2}} e^{1}, \quad \nabla_{e^{1}} e^{3}=e^{2}=\nabla_{e^{3}} e^{1}, \\
& \nabla_{e^{1}} e^{4}=-e^{5}=\nabla_{e^{4}} e^{1}, \quad \nabla_{e^{1}} e^{5}=e^{4}=\nabla_{e^{5}} e^{1}, \quad \nabla_{e^{2} e^{3}}=e^{1}=-\nabla_{e^{3}} e^{2}, \\
& \nabla_{e^{4}} e^{5}=e^{1}=-\nabla_{e^{5}} e^{4}
\end{aligned}
$$

and all other covariant derivatives vanish.

If now $A=a_{2} e^{2}+\cdots+a_{5} e^{5}$ (note. $A$ being $\pi$-related to a vector field on $\mathbf{C P}^{2}$ will now be equivalent to $a_{i}$ being constant),

$$
\begin{aligned}
& \nabla_{e^{1}} A=\left(e^{1}\left(a_{2}\right)+a_{3}\right) e^{2}+\left(e^{1}\left(a_{3}\right)-a_{2}\right) e^{3}+\left(e^{1}\left(a_{4}\right)+a_{5}\right) e^{4}+\left(e^{1}\left(a_{5}\right)-a_{4}\right) e^{5} \\
& \nabla_{e^{2}} A=e^{2}\left(a_{2}\right) e^{2}+e^{2}\left(a_{3}\right) e^{3}+e^{2}\left(a_{4}\right) e^{4}+e^{2}\left(a_{5}\right) e^{5}+a_{3} e^{1} \\
& \nabla_{e^{3}} A=e^{3}\left(a_{2}\right) e^{2}+e^{3}\left(a_{3}\right) e^{3}+e^{3}\left(a_{4}\right) e^{4}+e^{3}\left(a_{5}\right) e^{5}-a_{2} e^{1} \\
& \nabla_{e^{4}} A=e^{4}\left(a_{2}\right) e^{2}+e^{4}\left(a_{3}\right) e^{3}+e^{4}\left(a_{4}\right) e^{4}+e^{4}\left(a_{5}\right) e^{5}+a_{5} e^{1} \\
& \nabla_{e^{5}} A=e^{5}\left(a_{2}\right) e^{2}+e^{5}\left(a_{3}\right) e^{3}+e^{5}\left(a_{4}\right) e^{4}+e^{5}\left(a_{5}\right) e^{5}-a_{4} e^{1} .
\end{aligned}
$$

Then, we can compute the first variation: $F^{\prime}=\int_{M} 2(\operatorname{div}(J A)) d V=0$. Thus the Hopf fibration is a critical point of the volume functional, as claimed.

THEOREM 2.1. The Hopf fibration on the round $S^{5}$ is not a local minimum of the volume functional. 
Proof. Consider the second variation at the Hopf fibration, where $\xi=e^{1}$, $A=\mathscr{F}^{\prime}$, and $A^{\prime}=\mathscr{F}^{\prime \prime}$ :

$$
\begin{aligned}
F^{\prime \prime}=\int_{M} \frac{1}{4}[ & \sum\left\langle\nabla_{e^{j_{1}}} A^{\prime} \wedge \cdots \wedge \nabla_{e^{j_{\lambda}}} e^{1}\right. \\
& \left.+\cdots+\nabla_{e^{j_{1}}} e^{1} \wedge \cdots \wedge \nabla_{e^{j_{\lambda}}} A^{\prime}, \nabla_{e^{j_{1}}} e^{1} \wedge \cdots \wedge \nabla_{e^{j_{\lambda}}} e^{1}\right\rangle \\
& +\left|\nabla_{e^{j_{1}}} A \wedge \cdots \wedge \nabla_{e^{j_{\lambda}}} e^{1}+\cdots+\nabla_{e^{j_{1}}} e^{1} \wedge \cdots \wedge \nabla_{e^{j_{\lambda}}} A\right|^{2} \\
+ & 2\left\langle\nabla_{e^{j_{1}}} A \wedge \nabla_{e^{j_{2}}} A \wedge \cdots \wedge \nabla_{e^{j_{\lambda}}} e^{1}+\cdots\right. \\
+ & \left.\nabla_{e^{j_{1}}} e^{1} \wedge \cdots \wedge \nabla_{e^{j_{\lambda}-1}} A \wedge \nabla_{e^{j_{\lambda}}} A, \nabla_{e^{j_{1}}} e^{1} \wedge \cdots \wedge \nabla_{e^{j_{\lambda}}} e^{1}\right\rangle \\
& \left.\quad-4\left(-e^{2}\left(a_{3}\right)+e^{3}\left(a_{2}\right)-e^{4}\left(a_{5}\right)+e^{5}\left(a_{4}\right)\right)^{2}\right] d V
\end{aligned}
$$

where the sum is taken over $\lambda=1, \ldots, 4$, and $1 \leq j_{1}<\cdots<j_{\lambda} \leq 5$.

If now $A^{\prime}=b_{1} e^{1}+\cdots+b_{5} e^{5}$ (note: $A$ being $\pi$-related to a vector field on $\mathbf{C P}^{2}$ will now be equivalent to $a_{i}$ being constant on the fiber.),

$$
\begin{aligned}
\nabla_{e^{1}} A^{\prime}= & e^{1}\left(b_{1}\right) e^{1}+\left(e^{1}\left(b_{2}\right)+b_{3}\right) e^{2} \\
& +\left(e^{1}\left(b_{3}\right)-b_{2}\right) e^{3}+\left(e^{1}\left(b_{4}\right)+b_{5}\right) e^{4}+\left(e^{1}\left(b_{5}\right)-b_{4}\right) e^{5}, \\
\nabla_{e^{2}} A^{\prime}= & \left(e^{2}\left(b_{1}\right)+b_{3}\right) e^{1}+e^{2}\left(b_{2}\right) e^{2}+\left(e^{2}\left(b_{3}\right)-b_{1}\right) e^{3}+e^{2}\left(b_{4}\right) e^{4}+e^{2}\left(b_{5}\right) e^{5}, \\
\nabla_{e^{3}} A^{\prime}= & \left(e^{3}\left(b_{1}\right)-b_{2}\right) e^{1}+\left(e^{3}\left(b_{2}\right)+b_{1}\right) e^{2}+e^{3}\left(b_{3}\right) e^{3}+e^{3}\left(b_{4}\right) e^{4}+e^{3}\left(b_{5}\right) e^{5}, \\
\nabla_{e^{4}} A^{\prime}= & \left(e^{4}\left(b_{1}\right)+b_{5}\right) e^{1}+e^{4}\left(b_{2}\right) e^{2}+e^{4}\left(b_{3}\right) e^{3}+e^{4}\left(b_{4}\right) e^{4}+\left(e^{4}\left(b_{5}\right)-b_{1}\right) e^{5}, \\
\nabla_{e^{5}} A^{\prime}= & \left(e^{5}\left(b_{1}\right)-b_{4}\right) e^{1}+e^{5}\left(b_{2}\right) e^{2}+e^{5}\left(b_{3}\right) e^{3}+\left(e^{5}\left(b_{4}\right)+b_{1}\right) e^{4}+e^{5}\left(b_{5}\right) e^{5} .
\end{aligned}
$$

Then

$$
\begin{gathered}
F^{\prime \prime}=\int_{M}\left(8 b_{1}+2\left(-e^{2}\left(b_{3}\right)+e^{3}\left(b_{2}\right)-e^{4}\left(b_{5}\right)+e^{5}\left(b_{4}\right)\right)+2\left(a_{3}^{2}+a_{2}^{2}+a_{5}^{2}+a_{4}^{2}\right)\right. \\
+2\left(\left(e^{1}\left(a_{2}\right)+a_{3}\right)^{2}+\left(e^{1}\left(a_{3}\right)-a_{2}\right)^{2}+\left(e^{1}\left(a_{4}\right)+a_{5}\right)^{2}+\left(e^{1}\left(a_{5}\right)-a_{4}\right)^{2}\right) \\
+\left(e^{2}\left(a_{2}\right)+e^{3}\left(a_{3}\right)\right)^{2}+\left(e^{2}\left(a_{4}\right)+e^{5}\left(a_{3}\right)\right)^{2}+\left(e^{2}\left(a_{5}\right)-e^{4}\left(a_{3}\right)\right)^{2} \\
+\left(e^{3}\left(a_{4}\right)-e^{5}\left(a_{2}\right)\right)^{2}+\left(e^{3}\left(a_{5}\right)+e^{4}\left(a_{2}\right)\right)^{2}+\left(e^{4}\left(a_{4}\right)+e^{5}\left(a_{5}\right)\right)^{2} \\
\left.+\left(e^{2}\left(a_{3}\right)-e^{3}\left(a_{2}\right)+e^{4}\left(a_{5}\right)-e^{5}\left(a_{4}\right)\right)^{2}\right) d V .
\end{gathered}
$$

Now, $b_{1}=-|A|^{2}$, as can easily be seen, and the terms involving $b_{2}, \ldots, b_{5}$ are exact, so the integral becomes

$$
\begin{aligned}
F^{\prime \prime}=\int_{M}( & -6\left(a_{3}^{2}+a_{2}^{2}+a_{5}^{2}+a_{4}^{2}\right) \\
& +2\left(\left(e^{1}\left(a_{2}\right)+a_{3}\right)^{2}+\left(e^{1}\left(a_{3}\right)-a_{2}\right)^{2}+\left(e^{1}\left(a_{4}\right)+a_{5}\right)^{2}+\left(e^{1}\left(a_{5}\right)-a_{4}\right)^{2}\right) \\
& +\left(e^{2}\left(a_{2}\right)+e^{3}\left(a_{3}\right)\right)^{2}+\left(e^{2}\left(a_{4}\right)+e^{5}\left(a_{3}\right)\right)^{2}+\left(e^{2}\left(a_{5}\right)-e^{4}\left(a_{3}\right)\right)^{2} \\
& +\left(e^{3}\left(a_{4}\right)-e^{5}\left(a_{2}\right)\right)^{2}+\left(e^{3}\left(a_{5}\right)+e^{4}\left(a_{2}\right)\right)^{2}+\left(e^{4}\left(a_{4}\right)+e^{5}\left(a_{5}\right)\right)^{2} \\
& \left.\quad+\left(e^{2}\left(a_{3}\right)-e^{3}\left(a_{2}\right)+e^{4}\left(a_{5}\right)-e^{5}\left(a_{4}\right)\right)^{2}\right) d V
\end{aligned}
$$

Note that the terms involving $e^{i}\left(a_{j}\right), i>1$, are all nonnegative. Thus the contribution of the baselike derivatives will be nonnegative. However, recombining 
terms,

$$
\begin{aligned}
F^{\prime \prime}=\int_{M}( & -6\left(a_{3}^{2}+a_{2}^{2}+a_{5}^{2}+a_{4}^{2}\right) \\
& +2\left(\left(e^{1}\left(a_{2}\right)+a_{3}\right)^{2}+\left(e^{1}\left(a_{3}\right)-a_{2}\right)^{2}+\left(e^{1}\left(a_{4}\right)+a_{5}\right)^{2}+\left(e^{1}\left(a_{5}\right)-a_{4}\right)^{2}\right) \\
& +\left(e^{2}\left(a_{2}\right)+e^{3}\left(a_{3}\right)\right)^{2}+\left(e^{2}\left(a_{3}\right)-e^{3}\left(a_{2}\right)\right)^{2}+\left(e^{4}\left(a_{4}\right)+e^{5}\left(a_{5}\right)\right)^{2} \\
& +\left(e^{4}\left(a_{5}\right)-e^{5}\left(a_{4}\right)\right)^{2}+\left(e^{2}\left(a_{4}\right)+e^{3}\left(a_{5}\right)\right)^{2}+\left(e^{2}\left(a_{5}\right)-e^{3}\left(a_{4}\right)\right)^{2} \\
& +\left(e^{4}\left(a_{2}\right)+e^{5}\left(a_{3}\right)\right)^{2}+\left(e^{4}\left(a_{3}\right)-e^{5}\left(a_{2}\right)\right)^{2} \\
& +2\left[\left(e^{2}\left(a_{4}\right)-e^{4}\left(a_{2}\right)\right)\left(e^{5}\left(a_{3}\right)-e^{3}\left(a_{5}\right)\right)+\left(e^{2}\left(a_{5}\right)-e^{5}\left(a_{2}\right)\right)\right. \\
& \left.\left.\quad \cdot\left(e^{3}\left(a_{4}\right)-e^{4}\left(a_{3}\right)\right)+\left(e^{2}\left(a_{3}\right)-e^{3}\left(a_{2}\right)\right)\left(e^{4}\left(a_{5}\right)-e^{5}\left(a_{4}\right)\right)\right]\right) d V
\end{aligned}
$$

If $\alpha=\langle A, \cdot\rangle=a_{2} \theta^{2}+\cdots+a_{5} \theta^{5}$, where $\theta^{i}=\left\langle e^{i}, \cdot\right\rangle$, then, since $\left[e^{1}, e^{i}\right]=0$ for this framing, and $\left[e^{i}, e^{j}\right]=\lambda_{j}^{i} e^{1}, d \theta^{i}=0, i>1$; it follows that the last terms in the integrand are $2 d \alpha \wedge d \alpha \wedge d x=d(2 \alpha \wedge d \alpha \wedge d x)$, where $x$ is the fiber parameter. Thus,

$$
F^{\prime \prime}=\int_{M}\left(-6|\omega|^{2}+2\left|e^{1}(\omega)-i \omega\right|^{2}+4|\partial \omega|^{2}\right) d V,
$$

where $\omega=\left(a_{2}+i a_{3}, a_{4}+i a_{5}\right)$ and

$$
\begin{aligned}
|\partial \omega|^{2}= & \frac{1}{4}\left(\left(e^{2}\left(a_{2}\right)+e^{3}\left(a_{3}\right)\right)^{2}+\left(e^{2}\left(a_{3}\right)-e^{3}\left(a_{2}\right)\right)^{2}+\left(e^{4}\left(a_{4}\right)+e^{5}\left(a_{5}\right)\right)^{2}\right. \\
& +\left(e^{4}\left(a_{5}\right)-e^{5}\left(a_{4}\right)\right)^{2}+\left(e^{2}\left(a_{4}\right)+e^{3}\left(a_{5}\right)\right)^{2}+\left(e^{2}\left(a_{5}\right)-e^{3}\left(a_{4}\right)\right)^{2} \\
& \left.+\left(e^{4}\left(a_{2}\right)+e^{5}\left(a_{3}\right)\right)^{2}+\left(e^{4}\left(a_{3}\right)-e^{5}\left(a_{2}\right)\right)^{2}\right) .
\end{aligned}
$$

We can perform the Fourier series decomposition on the $\mathbf{C}^{2}$-valued function $\omega=\left(a_{2}+i a_{3}, a_{4}+i a_{5}\right)$, on each fiber. The $n$th Fourier coefficient will be identified with a section of $T_{*}\left(\mathbf{P}^{2}\right) \otimes O(-n)$. Letting $\omega=\sum \omega_{n} e^{i n x}$ and integrating over the fiber, then

$$
F^{\prime \prime}=4 \pi \int_{\mathbf{P}^{2}}\left(\sum_{n}\left(n^{2}-2 n-2\right)\left|\omega_{n}\right|^{2}+\left|{\overline{\partial \omega_{n}}}_{n}\right|^{2}\right) d V,
$$

where now $\bar{\omega}_{n} \in \Gamma\left(T^{*}\left(\mathbf{P}^{2}\right) \otimes O(n)\right.$, and $\bar{\partial}$ is the Deaubault operator. (The extra factor of $\frac{1}{2}$ comes from the pointwise norm of the $d \bar{z}$ terms.) This is clearly positive definite, except perhaps at $n=0,1,2$.

Now, the case $n=2$ will include the infinitesimal deformations of Hopf vector fields as a six-dimensional nullspace for the second variation. However, in contrast to the case for the three-sphere, these vector fields do not correspond to holomorphic sections of the appropriate twist of the cotangent bundle of $\mathbf{P}^{2}$. Rather, these fields correspond to the first positive eigenspace of $\bar{\square}$. There is a six (real)-dimensional space of holomorphic sections, however, on which the second variation is negativedefinite. Thus the Hopf fibration will not be area-minimizing on $S^{5}$. Similar results will likely hold for higher dimensions as well.

To show that there is a space on which $F^{\prime \prime}$ is negative, note that $H^{0}\left(\mathbf{P}^{2}, \Omega^{1}(2)\right) \simeq$ $\mathrm{C}^{3}[13]$. Let $\alpha$ be a nonzero holomorphic section of $\Omega^{1}(2), \bar{\alpha}=\omega$ will then be a section of $T_{*}\left(\mathrm{P}^{2}\right) \otimes O(-2)$. A vector field $A$ perpendicular to $\xi$ will then be defined by $\pi_{*} A=\omega$, where $\pi: S^{5} \times \mathbf{C} \rightarrow O(-2)$ is the map defining $O(-2)$ as an associated bundle to the principal bundle $S^{5} \rightarrow \mathbf{P}^{2}$. For this infinitesimal deformation $A$ of the Hopf fibration, $F^{\prime \prime}=8 \pi \int_{\mathbf{P}^{2}}-|\omega|^{2} d V<0$. 
ADDED IN PROOF. The author has recently been able to show that, in contrast to the situation on surfaces, the only homotopy class of flows on $S^{3}$ having a minimum-volume representative is the class containing the Hopf flows. Within any other homotopy class (they are given by $\pi_{3}\left(S^{2}\right)$ ), the infimum is again the volume of the Hopf flow, and so is not obtainable within the homotopy class. (This result is similar to theorems of Brian White, though in a slightly different context.) I would like to thank Sharon Pedersen for describing an example which inspired the construction of a minimizing sequence within an arbitrary homotopy class.

\section{REFERENCES}

1. M. Berger, Nonlinearity and functional analysis, Academic Press, New York, 1977.

2. I. Chavel and E. Feldman, The first eigenvalue of the Laplacian on manifolds of nonnegative curvature, Proc. Sympos. Pure Math., vol. 27, Amer. Math. Soc., Providence, R.I., 1975, pp. 351-353.

3. R. Escobales, Riemannian foliations of the rank-one symmetric spaces, Proc. Amer. Math. Soc. 95 (1985), 495-498.

4. J. Girbau, A. Haefliger and D. Sundararaman, On deformations of transverseley holomorphic foliations, J. Reine Angew. Math. 346 (1983), 122-147.

5. H. Gluck, Can space be filled by geodesics, and if so, how?, manuscript.

6. __ Dynamical behavior of geodesic fields, Global Theory of Dynamical Systems (Proc. Northwestern Univ., 1979), Lecture Notes in Math., vol. 819, Springer-Verlag, Berlin, Heidelberg, and New York, 1980, pp. 190-215.

7. H. Gluck and W. Ziller, On the volume of a unit vector field on the three-sphere, Comment. Math. Helv. 61 (1986), 177-192.

8. J. Heitsch, A cohomology for foliated manifolds, Comment. Math. Helv. 50 (1975), 197-218.

9. D. L. Johnson, Deformations of totally geodesic foliations, Geometry and Topology: Manifolds, Varieties (Proc. 1985 Georgia Topology Festival; C. McCrory and T. Shifrin, eds.), Marcel Dekker, 1986, pp. 167-178.

10.

11. D. L. Johnson and L. B. Whitt, Totally geodesic foliations, J. Differential Geom. 15 (1980), 225-235.

12. L. Nirenberg, VariatIonal and topological methods in nonlinear analysis, Bull. Amer. Math. Soc. (N.S.) 4 (1981), 276-302.

13. C. Okonek, M. Schneider, and H. Spindler, Vector bundles on complex projective spaces, Progress in Math. \# 3, Birkhäuser, Boston, Mass., 1980.

14. B. O'Neill, The fundamental equations of a submersion, Michigan Math. J. 13 (1966), 459-469.

15. S. Sasaki, On the differential geometry of tangent bundles of Riemannian manifolds, Tôhoku Math. J. 10 (1958), 338-354.

Department of Mathematics, Lehigh University, Christmas-Saucon Hall \#14, Bethlehem, PenNSylvania 18015 